\title{
Machine-learning analysis outperforms conventional statistical models and CT classification systems in predicting 6-month outcomes in pediatric patients sustaining traumatic brain injury
}

\author{
Andrew T. Hale, MS, ${ }^{1,2}$ David P. Stonko, MD, ${ }^{3}$ Amber Brown, BS, ${ }^{1}$ Jaims Lim, MD, ${ }^{1}$ \\ David J. Voce, MD, ${ }^{4}$ Stephen R. Gannon, BS, ${ }^{1}$ Truc M. Le, MD, ${ }^{5}$ and Chevis N. Shannon, DrPH ${ }^{1,4}$ \\ ${ }^{1}$ Surgical Outcomes Center for Kids, and ${ }^{5}$ Division of Pediatric Critical Care Medicine, Monroe Carell Jr. Children's Hospital \\ at Vanderbilt; ${ }^{2}$ Vanderbilt University School of Medicine, Medical Scientist Training Program; ${ }^{3}$ Vanderbilt University School of \\ Medicine; ${ }^{4}$ Department of Neurosurgery, Vanderbilt University Medical Center, Nashville, Tennessee
}

\begin{abstract}
OBJECTIVE Modern surgical planning and prognostication requires the most accurate outcomes data to practice evidence-based medicine. For clinicians treating children following traumatic brain injury (TBI) these data are severely lacking. The first aim of this study was to assess published CT classification systems in the authors' pediatric cohort. A pediatric-specific machine-learning algorithm called an artificial neural network (ANN) was then created that robustly outperformed traditional CT classification systems in predicting TBI outcomes in children.
\end{abstract}

METHODS The clinical records of children under the age of 18 who suffered a TBI and underwent head CT within 24 hours after TBI $(n=565)$ were retrospectively reviewed.

RESULTS "Favorable" outcome (alive with Glasgow Outcome Scale [GOS] score $\geq 4$ at 6 months postinjury, $n=533$ ) and "unfavorable" outcome (death at 6 months or GOS score $\leq 3$ at 6 months postinjury, $n=32$ ) were used as the primary outcomes. The area under the receiver operating characteristic (ROC) curve (AUC) was used to delineate the strength of each CT grading system in predicting survival (Helsinki, 0.814; Rotterdam, 0.838; and Marshall, 0.781). The AUC for CT score in predicting GOS score $\leq 3$, a measure of overall functionality, was similarly predictive (Helsinki, 0.717; Rotterdam, 0.748; and Marshall, 0.663 ). An ANN was then constructed that was able to predict 6 -month outcomes with profound accuracy (AUC $=0.9462 \pm 0.0422$ ).

CONCLUSIONS This study showed that machine-learning can be leveraged to more accurately predict TBI outcomes in children.

https://thejns.org/doi/abs/10.3171/2018.8.FOCUS17773

KEYWORDS neurotrauma; traumatic brain injury; artificial neural network

$\mathrm{D}$ IFFUSE axonal injury, space-occupying hemorrhages, cerebral edema, or a combination of these features are manifestations of traumatic brain injury (TBI). However, the etiologies of TBI are diverse and heterogeneous. Therefore, robust predictive models are needed to stratify pertinent variables affecting clinical outcomes. In this study we explore a number of established scoring systems as well as introduce a novel machine-learning approach to predictive modeling in TBI.
Classically, the Glasgow Coma Scale (GCS) score is used to categorize the severity of TBIs into one of three categories: mild, moderate, or severe. However, reproducible assessment of GCS and inter-rater reliability determining GCS is highly variable. . $^{2,711}$ One study determined the GCS score for severe TBIs in pediatrics should be $<5$, however, confounding factors in assigning a GCS score, such as alcohol intoxication, suggest that GCS score cannot be used as a sole predictor. ${ }^{9}$ Furthermore, evaluating

ABBREVIATIONS ANN = artificial neural network; $A U C=$ area under the curve; $\mathrm{DI}=$ diffuse injury; $E D H=$ epidural hematoma; GCS = Glasgow Coma Scale; $\mathrm{GOS}=$ Glasgow Outcome Scale; ICH = intracranial hemorrhage; IVH = intraventricular hemorrhage; ROC = receiver operating characteristic; $\mathrm{SAH}=$ subarachnoid hemorrhage; $\mathrm{SDH}=$ subdural hemorrhage; $\mathrm{TBI}=$ traumatic brain injury; $\mathrm{tSAH}=$ traumatic $\mathrm{SAH}$. ACCOMPANYING EDITORIAL DOI: 10.3171/2018.8.FOCUS18438.

SUBMITTED December 18, 2017. ACCEPTED August 15, 2018.

INCLUDE WHEN CITING DOI: 10.3171/2018.8.FOCUS17773. 
response and verbal communication in young children is not feasible as their developmental stage does not facilitate accurate assessment. Thus, utilizing CT to identify objective structural deformities may provide a more accurate way to access severity of injury alongside clinical evaluation. Therefore, we aimed to evaluate previously established CT-based classification systems in the adult population in predicting morbidity and mortality after TBI in a large cohort of children.

The Marshall scale was the first CT-based classification system used to assess TBI. ${ }^{10}$ The Marshall score is composed of three variables: presence of mass lesion, midline shift, and status of the perimesencephalic cisterns. The Marshall score provides descriptive and predictive values, but the narrow scope of pathology that is incorporated into the scoring system limits its broad applicability to clinical practice. Subjective assessments and the accuracy of the measured lesions are also important limitations to the Marshall scoring system. Next, the Rotterdam CT scoring system was created in response to the Marshall system's limitations, thereby taking subarachnoid hemorrhage (SAH) into account. ${ }^{8}$ One of the biggest limitations of the Rotterdam CT scoring system is that it does not differentiate between the type and size of the mass lesion. The Helsinki system uses a different calculation in comparison to the other CT scoring systems; the presence of an epidural hematoma (EDH) yields negative points, while the absence of an EDH under the Rotterdam system yields positive points. ${ }^{13}$ In adults, the Helsinki score is superior in predicting outcome in comparison to Rotterdam CT or Marshall CT scores when used as a standalone predictive method. ${ }^{13}$ However, these classification systems do not quantitatively rely on existing data. This study aims to provide a comparison of the three CT scoring systems-Marshall, Rotterdam, and Helsinki-and their ability to predict survival and functional outcomes after TBI in children. Next, we propose a machine-learning algorithm-based model of outcomes in patients with TBI that vastly outperforms existing classification schema.

Creation of a mathematical model that can accurately predict long-term outcomes post-TBI can assist clinicians involved in all aspects of the child's care. Compared to traditional regression models, machine-learning algorithms, such as artificial neural networks (ANNs), can provide very strong predictive data despite a relatively low number of poor outcomes. ${ }^{12,16,19}$ Compared to limitations of degrees of freedom in multivariate analysis models, ANNs can robustly predict binary outcomes given a sufficient number of total data presented to the algorithm. ANN models can be trained and refined, randomly assigning relative weight to each input variable to construct the most robust prediction. 3,5,6 Although ANNs are considered "black-box" computational models, their value in clinical medicine has enormous potential to engage in evidence-based medical practice because they can be trained on new patient information. ANN models also benefit from internal validation and testing and tend to have much stronger predictive ability of binary outcomes compared to multivariate regression modeling. Thus, ANNs have been designed to use patient factors and disease characteristics (such as histopathologi- cal features from a tumor biopsy or demographic information) to predict outcomes in various clinical models, including TBI, postsurgical outcomes, and complications from surgery. ${ }^{4,6,14,17,18}$ Previous traditional statistical models have failed in their inflexibility and inability to change based on the data that is presented. Therefore, we aimed to create a model that can predict outcomes using binary and continuous variables allowing for real-time clinical utilization, resulting in greater accuracy and higher predictive value in children sustaining TBI.

\section{Methods \\ Data Collection}

Using ICD-9 and ICD-10 codes, electronic medical records of 565 patients ( $0-18$ years of age) treated for mild to severe TBI between 2006 and 2013 at Monroe Carell Jr. Children's Hospital at Vanderbilt University were reviewed. Patients who did not receive a CT scan within the first 24 hours of admissions were excluded from the study. Patients who suffered fatal injuries on arrival were excluded from analysis. Upon admission, a GCS score was collected by an emergency medicine physician or neurosurgeon. Alternatively, a postgraduate year-4 neurosurgery resident (D.J.V.) retrospectively calculated the GCS score based on the documented physical examination findings if the admitting GCS score was unavailable. The Glasgow Outcome Scale (GOS) score was calculated based on documentation by any medical provider 6 months after TBI. Patients who were lost to follow up before the 6-month assessment but discharged in stable condition following initial injury were assumed to be alive at 6 months after trauma with a GOS score of 5. Admitting blood glucose levels and hemoglobin results were obtained by retrospective chart review. A board-certified radiologist interpreted all CT scans used in this study and hemorrhage (intraventricular, intracerebral, and subarachnoid), presence of midline shift above $5 \mathrm{~mm}$, cistern integrity, hematoma (epidural and subdural), and mass lesions $>25 \mathrm{~mm}^{3}$ was documented. CT classification systems-Marshall, Helsinki, and Rotterdam-were applied as previously described. ${ }^{13}$

\section{Statistical Analysis}

Descriptive statistics were used to evaluate our normally distributed cohort. Multivariable logistic regression was used to assess the association between Helsinki, Rotterdam, and Marshall CT grading scores and survival at 6 months posttrauma with the adjustment of age. The area under the receiver operating characteristic (ROC) curve (AUC) was used to evaluate the ability to discriminate the 6-month posttrauma survival status of patients. Statistical analysis was conducted using $\mathrm{R}$ statistical program (version 3.3.1) and statistical significance was set a priori at $\mathrm{p}<0.05$.

\section{Artificial Neural Network}

We utilized the MATLAB Neural Network Toolbox to construct an ANN that classified patients into two groups, either "favorable 6-month outcomes" or "unfavorable 6-month outcomes," where unfavorable outcome was defined as death or GOS score $\leq 3$. This ANN is a two-layer 
TABLE 1. Baseline patient characteristics

\begin{tabular}{|c|c|c|c|c|}
\hline Variable & All Patients $(n=565)$ & Favorable Outcome $(n=533)$ & Unfavorable Outcome $(n=32)$ & $p$ Value \\
\hline Mean GCS score \pm SD & $12.90 \pm 3.88$ & $13.34 \pm 3.37$ & $5.78 \pm 4.62$ & $<0.001$ \\
\hline Mean motor score $\pm \mathrm{SD}^{*}$ & $5.46 \pm 1.34$ & $5.62 \pm 1.09$ & $2.87 \pm 2.19$ & $<0.001$ \\
\hline Eye opening, n (\%) & & & & $<0.001$ \\
\hline Spontaneously & $418(74)$ & $410(77)$ & $9(28)$ & - \\
\hline To speech & $51(9)$ & $48(9)$ & $0(0)$ & - \\
\hline To pain & $17(3)$ & $16(3)$ & $1(3)$ & - \\
\hline No response & 79 (14) & $59(11)$ & $22(69)$ & - \\
\hline Mean verbal response $\pm \mathrm{SD}^{*}$ & $4.21 \pm 1.49$ & $4.35 \pm 1.36$ & $1.93 \pm 1.66$ & $<0.001$ \\
\hline Pupillary light reaction, n (\%) & & & & $<0.001$ \\
\hline Both eyes & $525(93)$ & $506(95)$ & $20(63)$ & - \\
\hline 1 reacts & $34(6)$ & $27(5)$ & $11(34)$ & - \\
\hline None reacts & $6(1)$ & $0(0)$ & $1(3)$ & - \\
\hline Mean glucose level \pm SD, mg/dl & $140.3 \pm 67.2$ & $134.9 \pm 53.4$ & $210.0 \pm 140.6$ & $<0.001$ \\
\hline Mean hemoglobin level \pm SD, g/dl & $12.04 \pm 4.76$ & $12.15 \pm 4.83$ & $10.50 \pm 3.35$ & 0.03 \\
\hline Mass lesions, $\mathrm{n}(\%)$ & & & & - \\
\hline $\mathrm{SDH}$ & $185(33)$ & $168(32)$ & $17(53)$ & 0.015 \\
\hline $\mathrm{EDH}$ & $74(13)$ & $69(13)$ & $5(16)$ & 0.698 \\
\hline $\mathrm{ICH}$ & $68(12)$ & $60(11)$ & $5(16)$ & 0.024 \\
\hline $\mathrm{IVH}$ & $15(3)$ & $10(2)$ & $5(16)$ & 0.001 \\
\hline tSAH, n (\%) & $124(22)$ & $16(3)$ & $10(31)$ & 0.001 \\
\hline Cisterns, n (\%) & & & & 0.001 \\
\hline Normal & $541(96)$ & $514(96)$ & $27(84)$ & - \\
\hline Compressed & $6(1)$ & $4(1)$ & $2(6)$ & - \\
\hline Obliterated & $7(1)$ & $4(1)$ & $3(9)$ & - \\
\hline Midline shift >5 mm, $\mathrm{n}(\%)$ & $11(2)$ & $8(2)$ & $3(9)$ & 0.002 \\
\hline
\end{tabular}

${ }^{*} n=529$.

feed-forward network, with 10 sigmoid hidden and softmax output neurons. The network was trained with scaled conjugate gradient method backpropagation. Seventy percent of the data set was allocated for training, $15 \%$ for validation, and $15 \%$ for testing. Our approach was performed according to previous reports. ${ }^{1,6,12,14}$

This network was trained 100 times using different, random weights and data partitions. That is, we initialized the ANN with the data architecture, except that upon each run new randomized weights were calculated for each connection in the network. Next, the complete data set was partitioned randomly where each initialization of the model received a new, random partition. The model was then trained, and ROC curves and AUC was computed by the trapezoid method. Thus, we collected 100 ROCs and 100 corresponding AUCs. Using these, we computed the mean and standard deviation AUC for model training. All mathematical and graphical analysis was done using offline MATLAB R2016b (version 9.1.0.441655) with an academic license on a 64-bit MacBook Pro running version 10.11.6.

\section{Results}

In a 7-year time period, 565 children sustained a TBI and met inclusion criteria for this study. The charts of 565 patients were retrospectively reviewed and represented here. Five hundred thirty-three patients were determined to have a favorable outcome (alive with GOS score $\geq 4$ ) and 32 patients had an unfavorable outcome (death at 6 months or GOS score $\leq 3$ ). Baseline patient characteristics are shown in Table 1. The average patient age was 6.81 \pm 5.57 years, and there was no significant difference in age between patients with unfavorable and favorable outcomes $(p=0.63)$. Patients with unfavorable outcomes had significantly higher blood glucose levels at presentation $(210 \pm 140.6 \mathrm{mg} / \mathrm{dl})$ compared to patients with favorable outcomes $(134.9 \pm 53.9 \mathrm{mg} / \mathrm{dl} ; \mathrm{p}<0.001)$. There was also a significant difference in hemoglobin levels at admission between patients with favorable versus unfavorable outcomes $(p=0.03)$. As a positive control, GCS was evaluated based on clinical documentation at the time of admission. Patients with favorable outcomes were found to have an average GCS score of $13.34 \pm 3.37$, while patients with unfavorable outcomes were found to have a score of 5.78 $\pm 4.62(\mathrm{p}<0.001)$. Patients with a favorable outcome were found to have a higher pupillary response, motor response, and verbal response in comparison to patients with unfavorable outcome $(\mathrm{p}<0.001)$.

Patient CT characteristics are shown in Table 2. Predictably, subdural hemorrhage (SDH), intracranial hemorrhage (ICH), intraventricular hemorrhage (IVH), trau- 
TABLE 2. Baseline pediatric CT classification system scores

\begin{tabular}{ccccc}
\hline $\begin{array}{c}\text { CT Classification } \\
\text { System }\end{array}$ & $\begin{array}{c}\text { All } \\
\text { Patients }\end{array}$ & $\begin{array}{c}\text { Favorable } \\
\text { Outcome }\end{array}$ & $\begin{array}{c}\text { Unfavorable } \\
\text { Outcome }\end{array}$ & $\begin{array}{c}\mathrm{p} \\
\text { Value }\end{array}$ \\
\hline $\begin{array}{c}\text { Marshall score, } \\
\mathrm{n}(\%)\end{array}$ & & & & $<0.001$ \\
\hline DI I (1) & 198 & $192(97)$ & $6(3)$ & - \\
\hline DI II (2) & 351 & $331(94)$ & $20(6)$ & - \\
\hline DI III (3) & 6 & $2(33)$ & $4(67)$ & - \\
\hline DI IV (4) & 6 & 5 & 1 & - \\
\hline EML/NEML & 1 & 0 & 1 & - \\
\hline $\begin{array}{c}\text { Mean Rotterdam } \\
\text { score } \pm \text { SD }\end{array}$ & $1.67 \pm 0.87$ & $1.61 \pm 0.77$ & $2.63 \pm 1.58$ & $<0.001$ \\
\hline $\begin{array}{c}\text { Mean Helsinki } \\
\text { score } \pm \text { SD }\end{array}$ & $2.60 \pm 1.78$ & $2.51 \pm 1.68$ & $4.09 \pm 2.63$ & $<0.001$ \\
\hline
\end{tabular}

$\mathrm{EML}=$ evacuated mass lesion; $\mathrm{NEML}=$ nonevacuated mass lesion.

matic SAH (tSAH), integrity of the cisterns, and presence of midline shift were all significantly associated with unfavorable outcomes. However, EDH was not predictive of unfavorable outcome $(p=0.698)$. Among the total cohort, patients were classified most frequently as diffuse injury (DI) II using the Marshall CT classification ( $p<0.001$, Table 2). Patients with an unfavorable outcome were found to have a significantly higher Rotterdam CT score and Helsinki score $(2.63 \pm 1.58$ and $4.09 \pm 2.63$, respectively) in comparison to patients with favorable outcomes $(1.61 \pm$ 0.77 and $2.51 \pm 1.68$, respectively; $\mathrm{p}<0.001$; Table 2 ).

Marshall, Rotterdam, and Helsinki scores were individually associated with unfavorable outcomes (Table 2). Comparison of the predictive value using AUC for each CT classification system in predicting 6-month unfavorable outcome and 6-month mortality is shown in Table 3. GCS showed the highest predictive ability $\left(\mathrm{AUC}_{\text {unfavorable }}\right.$ $=0.855, \mathrm{AUC}_{\text {mortality }}=0.92$ ), followed by Rotterdam CT $\left(\mathrm{AUC}_{\text {unfavorable }}=0.748, \mathrm{AUC}_{\text {mortality }}=0.838\right)$, Helsinki $\left(\mathrm{AUC}_{\text {unfavorable }}=0.717, \mathrm{AUC}_{\text {mortality }}=0.814\right)$, and then Marshall CT $\left(\mathrm{AUC}_{\text {unfavorable }}=0.663, \mathrm{AUC}_{\text {mortality }}=0.781\right)$. These data suggest that $\mathrm{CT}$ classification systems can be applied to pediatric patients, but do not mirror results seen in the adult population.

Univariate logistic regression analysis was used to identify clinical variables (GCS score, pupillary light reaction, glucose, and hemoglobin) and features seen on CT (SDH, ICH, IVH, cistern integrity, and midline shift) that were statistically significant for predicting unfavorable outcomes (deceased or have a GOS score $\leq 3$ ) at 6 months postinjury (Table 1). Using these 9 variables as inputs, we constructed an ANN that can predict whether a patient will have a favorable or unfavorable outcome at 6 months postinjury. A graphical, simplified representation of this neural network is given in Fig. 1.

The network was trained using the algorithm (outlined in Fig. 1) 100 times with different, randomly generated initial weights and partitions for each input variable. ANNs work by randomly assigning weights to each given input (in this case, the variables listed in Fig. 1), and are used to generate the most accurate predictive model with
TABLE 3. Adult versus pediatric CT-based classification of 6-month outcomes after TBI

\begin{tabular}{ccc}
\hline & \multicolumn{2}{c}{ AUC } \\
\cline { 2 - 3 } Outcome Variable & $\begin{array}{c}\text { Adult } \\
\text { (Raj et al., 2014) }\end{array}$ & $\begin{array}{c}\text { Pediatric } \\
\text { (current study) }\end{array}$ \\
\hline $\begin{array}{c}\text { 6-month unfavorable outcome } \\
\text { (GOS score } \leq 3)\end{array}$ & \\
\hline Marshall CT & 0.632 & 0.663 \\
\hline Rotterdam CT & 0.682 & 0.748 \\
\hline Helsinki CT & 0.750 & 0.717 \\
\hline GCS score & Not reported & 0.855 \\
\hline 6-month mortality & & \\
\hline Marshall CT & 0.635 & 0.781 \\
\hline Rotterdam CT & 0.699 & 0.838 \\
\hline Helsinki CT & 0.744 & 0.814 \\
\hline GCS score & Not reported & 0.920 \\
\hline
\end{tabular}

the data that is provided during training. Over these 100 trainings and testing runs of the model, the mean $( \pm 95 \%$ confidence interval) AUC for the "All ROC" curve was $0.9462 \pm 0.0422$ (maximum AUC $=0.9774$ over 100 trainings; Fig. 2). Therefore, the average training of the model provided an AUC of 0.9462 , with some small variation depending upon the initial random weights and the data chosen in each partition. Of the 100 final models generated, the highest AUC was 0.9774.

\section{Discussion}

In this study we evaluated the use of the Marshall, Rotterdam, and Helsinki CT classification systems as clinical predictors of survival and outcome in pediatric patients with TBI. Next, we constructed a machine-learning algorithm (ANN) that was able to outperform these traditional classification systems through both inclusion of clinical and radiographic data as well as implementation of a novel statistical tool. Numerous studies have explored the utilization of CT classification systems and GCS scores in evaluating outcomes following TBI in adults. However, this is the first study detailing CT-based grading systems for evaluation of TBI in the pediatric population. In our cohort, all three CT-based grading systems (Marshall, Helsinki, and Rotterdam) had stronger predictive value for 6-month morbidity and mortality compared to reported results in adults. Contrary to results in adults, the Rotterdam scoring system was the most robust model in our study population (Table 3 ).

Next, we present the first description of an ANN in predicting outcomes post-TBI in a pediatric population. Our data confirm the potential clinical utility of ANNs and provide a rationale for their adoption in medical practice. We constructed an ANN incorporating the presence of injurious findings on CT as well as clinical parameters that were associated with worse clinical outcomes (mortality and GCS score $\leq 3$ ) based on univariate analyses. The power in this study is simplifying clinical prognostication based on a wide array of clinical findings that have 
Input Variables

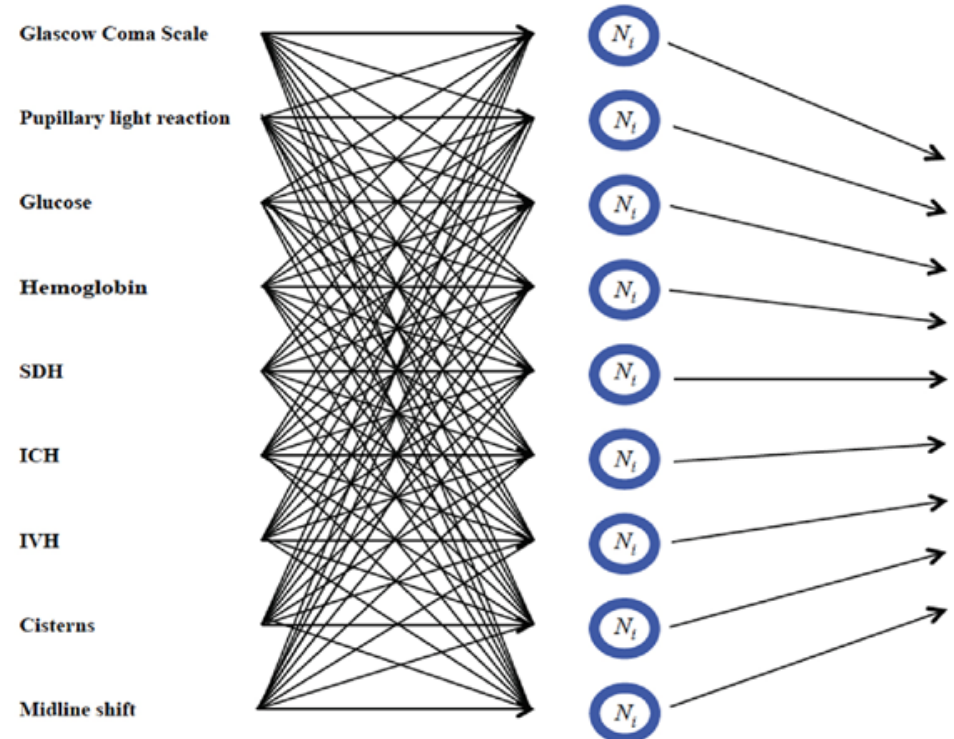

\section{Target Layer}

\section{Outcome: Favorable vs. Unfavorable}

FIG. 1. Representation showing how we created an ANN using 9 variables: 1) GCS score; 2) pupillary light reaction; 3) glucose at presentation; 4) hemoglobin at presentation; 5) $\mathrm{SDH}$; 6) ICH; 7) IVH; 8) integrity of the cisterns; and 9) presence of midline shift. The aforementioned 9 variables were all found to be statistically associated with poor outcomes at 6 months using univariate analysis (Table 1), providing the rationale to include them in the model. Outcome was determined to be favorable if the patient had a GOS score of 4 or 5 . Unfavorable outcome was defined as death or GOS score $\leq 3$.

been validated in our cohort. The basic principle of an ANN is its construct of multiple analytical layers made up of interconnected nodes with output (outcome) and input nodes given relative correlates (i.e., clinical factors). ${ }^{12}$ ANNs have been used extensively in the mathematical literature, however, their use in and translation to clinical medicine has been a relatively new idea. ${ }^{13-15}$ Widespread adoption of ANNs has been limited by the complex computational methods needed to construct a model de novo. However, we argue that incorporating ANNs into an online calculator, similar to calculating atherosclerotic cardiovascular disease risk score in predicting the likelihood of experiencing an adverse cardiac event, can usher in scientifically rigorous and complex mathematical algorithms, while simultaneously making these models practical for every physician to learn and use. Most importantly, accurate outcome prediction tools can provide real-time, data-driven information to families at the time of injury.

The use of ANNs in the TBI literature has been presented previously by Penny and Frost. ${ }^{12}$ Interestingly, their cohort was extremely large (16,956 patients), correlating with an AUC of 0.896 . However, our model better predicted poor outcomes with many fewer patients (565) and with greater accuracy $(\mathrm{AUC}=0.9462 \pm 0.0422)$. We note here that while our maximum AUC was quite good (maximum AUC $=0.9774$ ), our model's ability to discriminate did vary slightly based on the random initialization of the weights and data selected for each partition $(95 \%$ CI 0.0422). This incomplete robustness to randomization indicates that with a larger data set we may be able
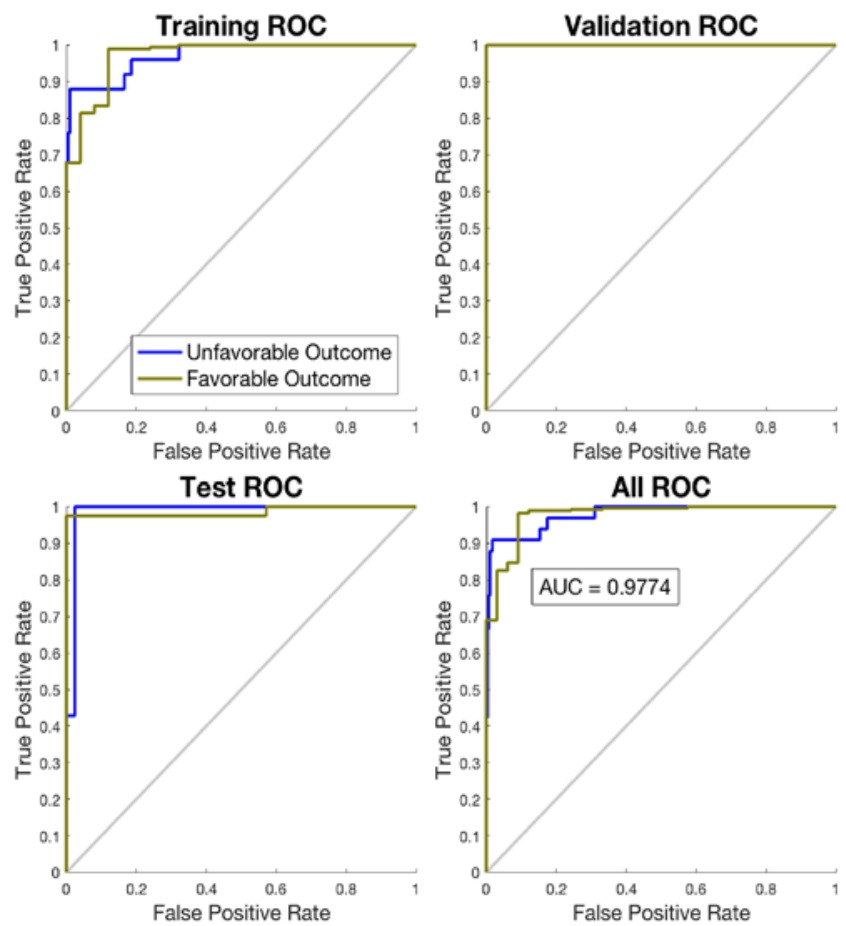

FIG. 2. ROC curves from a training of the neural network, which was very predictive. The blue line corresponds to the model's true positive versus false positive rate for predicting an unfavorable outcome. The brown line is analogous for favorable outcomes. The AUC for this representative training was 0.9774 for the "All ROC" curve. 
to further improve predictability with this methodology. We attribute the differences between our model and the aforementioned study to more rigorous selection of input variables, combining objective findings on $\mathrm{CT}$ as well as clinical assessment with both discrete and continuous variables in the input nodes. Furthermore, these authors only included patients who underwent surgery for treatment of TBI, whereas our cohort was a more accurate epidemiological cohort of children presenting to the emergency department with a wide spectrum of injuries. Shi et al. constructed an ANN to predict outcomes post-TBI using inpatient data only. ${ }^{15}$ Again, our model outperformed their predictive indexes and included data from patients in the emergency department, before the decision to admit or transfer to the intensive care unit. ANNs have also been used to predict outcomes in other neurosurgical conditions. Thus, our group and others have validated the superiority of ANNs to logistic regression models in multiple disease states.

With this model in hand, a clinician could utilize simple peri-injury data to predict the outcome and prognosis for patients presenting with TBI. The clinician could now more accurately plan follow-up management and early intervention therapy based on these data. Thus, adoption of this predictive model has the power to improve patient outcomes by providing the clinician with more accurate outcomes data at the time of injury. Thus, it could be imagined that this tool could be used to identify patients most at risk for adverse outcomes and triage them appropriately. The power of the ANN we presented here is the large number of variables the model is able to handle computationally, allowing for greater discrimination and resolution of outcome predictions.

However, our analysis is not without limitations. Our study was retrospective and performed at a single tertiary care center. Thus, a future aim is to conduct a prospective trial on utilization of this model and its influence on clinical-decision making and potentially induce early interventions to improve outcomes in these children. We imagine this to include analysis of prospectively collected data as well as allowing physicians to have access to this algorithm in real-time. We could then track outcomes of patients treated by providers who had the knowledge of the outcomes prediction generated from the ANN versus those who did not. Furthermore, ANNs are relatively new in the clinical outcomes literature, and the mathematics behind the analysis is conceptually difficult for the layman to abstract. For this reason, ANNs are often viewed as a "black box," where the average user provides input data and receives a prediction, often without understanding how the prediction is being generated. This is casually compared to conventional statistics in which, for example, a list of odds ratios and multivariate analysis can provide even nonstatisticians with an understanding of where the basis of a prediction lies. Sometimes, because the computational sophistication of a neural network is harder to intuit than a statistical measure, this is viewed as a weakness of neural networks. However, when the goal is to provide the most accurate prediction, a perfect understanding of the mechanism by which the prediction is being generated is often considered a reasonable sacrifice.

\section{Conclusions}

In this study we create a powerful model using an ANN that can readily predict outcomes of children with TBI. We believe that adoption of this model can greatly improve outcome predictions in evaluation of these injuries. As computer and smart phone access increases globally and electronic medical records become more sophisticated, evidence-based prediction software, akin to the data presented here, are poised to become ubiquitous in the modern healthcare landscape. This is first clinical prediction tool using an ANN that predicts 6-month post-TBI outcomes in children, and we demonstrate this tool's superior accuracy.

\section{References}

1. Baxt WG: Application of artificial neural networks to clinical medicine. Lancet 346:1135-1138, 1995

2. Bledsoe BE, Casey MJ, Feldman J, Johnson L, Diel S, Forred W, et al: Glasgow Coma Scale scoring is often inaccurate. Prehosp Disaster Med 30:46-53, 2015

3. Buscema PM, Massini G, Maurelli G: Artificial neural networks: an overview and their use in the analysis of the AMPHORA-3 dataset. Subst Use Misuse 49:1555-1568, 2014

4. Cross SS, Harrison RF, Sanders DS: Supporting decisions in clinical medicine: neural networks in lower gastrointestinal haemorrhage. Lancet 362:1250-1251, 2003

5. Forsström JJ, Dalton KJ: Artificial neural networks for decision support in clinical medicine. Ann Med 27:509-517, 1995

6. Gholipour C, Rahim F, Fakhree A, Ziapour B: Using an artificial neural networks (ANNs) model for prediction of intensive care unit (ICU) outcome and length of stay at hospital in traumatic patients. J Clin Diagn Res 9:OC19-OC23, 2015

7. Jamal A, Sankhyan N, Jayashree M, Singhi S, Singhi P: Full Outline of Unresponsiveness score and the Glasgow Coma Scale in prediction of pediatric coma. World J Emerg Med 8:55-60, 2017

8. Maas AI, Hukkelhoven CW, Marshall LF, Steyerberg EW: Prediction of outcome in traumatic brain injury with computed tomographic characteristics: a comparison between the computed tomographic classification and combinations of computed tomographic predictors. Neurosurgery 57:11731182,2005

9. Marmarou A, Lu J, Butcher I, McHugh GS, Murray GD, Steyerberg EW, et al: Prognostic value of the Glasgow Coma Scale and pupil reactivity in traumatic brain injury assessed pre-hospital and on enrollment: an IMPACT analysis. J Neurotrauma 24:270-280, 2007

10. Marshall LF, Marshall SB, Klauber MR, Van Berkum Clark M, Eisenberg H, Jane JA, et al: The diagnosis of head injury requires a classification based on computed axial tomography. J Neurotrauma 9 (Suppl 1):S287-S292, 1992

11. Momenyan S, Mousavi SM, Dadkhahtehrani T, Sarvi F, Heidarifar R, Kabiri F, et al: Predictive validity and interrater reliability of the Persian version of full outline of unresponsiveness among unconscious patients with traumatic brain injury in an intensive care unit. Neurocrit Care 27:229-236, 2017

12. Penny W, Frost D: Neural networks in clinical medicine. Med Decis Making 16:386-398, 1996

13. Raj R, Siironen J, Skrifvars MB, Hernesniemi J, Kivisaari R: Predicting outcome in traumatic brain injury: development of a novel computerized tomography classification system (Helsinki computerized tomography score). Neurosurgery 75:632-647, 2014

14. Rughani AI, Dumont TM, Lu Z, Bongard J, Horgan MA, 
Penar PL, et al: Use of an artificial neural network to predict head injury outcome. J Neurosurg 113:585-590, 2010

15. Shi HY, Hwang SL, Lee KT, Lin CL: In-hospital mortality after traumatic brain injury surgery: a nationwide populationbased comparison of mortality predictors used in artificial neural network and logistic regression models. J Neurosurg 118:746-752, 2013

16. Walczak S: Artificial neural network medical decision support tool: predicting transfusion requirements of ER patients. IEEE Trans Inf Technol Biomed 9:468-474, 2005

17. Wise ES, Stonko DP, Glaser ZA, Garcia KL, Huang JJ, Kim JS, et al: Prediction of prolonged ventilation after coronary artery bypass grafting: data from an artificial neural network. Heart Surg Forum 20:E007-E014, 2017

18. Yoldaş Ö, Tez M, Karaca T: Artificial neural networks in the diagnosis of acute appendicitis. Am J Emerg Med 30:12451247,2012

19. Zou J, Han Y, So SS: Overview of artificial neural networks. Methods Mol Biol 458:15-23, 2008

\section{Disclosures}

The authors report no conflict of interest concerning the materials or methods used in this study or the findings specified in this paper.

\section{Author Contributions}

Conception and design: Hale. Acquisition of data: Hale, Brown, Lim, Voce. Analysis and interpretation of data: Hale, Stonko. Drafting the article: Hale, Stonko. Critically revising the article: Hale. Reviewed submitted version of manuscript: Hale, Brown, Lim, Voce, Gannon, Le, Shannon. Approved the final version of the manuscript on behalf of all authors: Hale. Statistical analysis: Stonko. Administrative/technical/material support: Gannon. Study supervision: Le, Shannon.

\section{Correspondence}

Andrew T. Hale: Vanderbilt Children's Hospital, Nashville, TN. andrew.hale@vanderbilt.edu. 